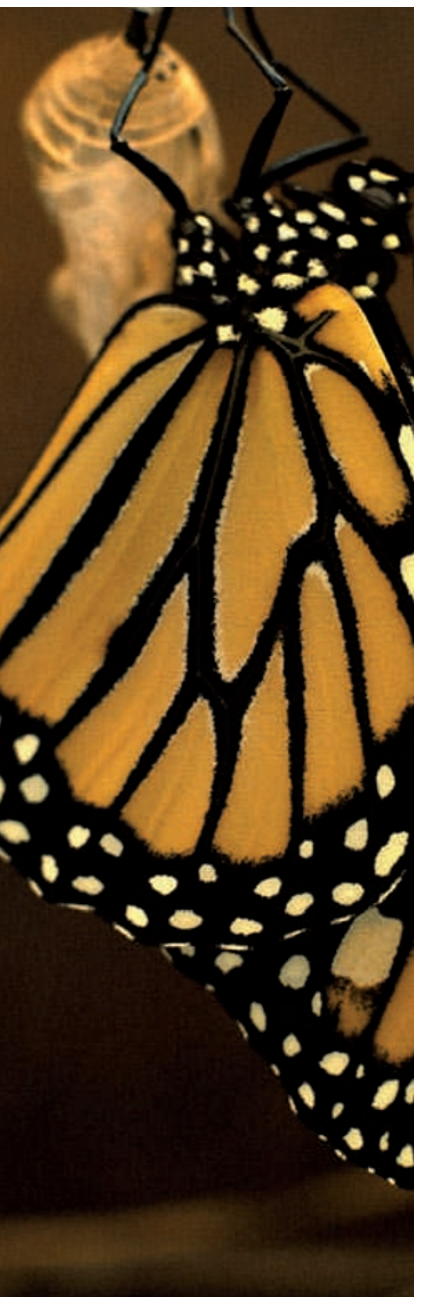

\title{
A metamorphosis in synthesis
}

If your aim is to understand functional regulatory elements in genomes or to use them to synthesize new genetic circuits, there is good news: no more need to bother with the tedious work of checking the effects of each possible mutation in a promoter, one base at a time. A new technique by Patwardhan et al. will allow labs to test all mutations at once, given access to a number of ultra-high-throughput technologies.

The authors started with a method published in 2004 by Cleary et al. to synthesize a massive number of oligonucleotides in parallel and then release the oligos into solution. In the new paper, the clever design of the oligos included three crucial elements: a promoter and transcriptional start site to be tested, featuring nucleotide substitutions at each position; flanking sequences of native DNA around this promoter; and a barcode unique to each oligo. Once in solution, the oligos were subjected to PCR along their entire length and then split into fractions. One fraction was used in an in vitro transcription reaction to make RNA from the promoter, and the RNA was then converted to DNA using RT-PCR. The second fraction was amplified using PCR only. Massively parallel sequencing was then carried out on the output of both reactions, and quantitative comparison between the RNA barcode and the DNA barcode was used to give a measure of promoter effectiveness. Obviously, several controls must also be included to take into account the varying efficiencies of the reactions.

To test this method, the authors generated all possible singlenucleotide substitutions and some double-nucleotide changes in three different and well-studied bacteriophage promoters: T3, T7 and Sp6. Starting from a pool of 12,972 different template oligos, they produced a complete picture of how each possible nucleotide at each position from -23 to +12 around the transcriptional start site changes transcriptional efficiency. As expected, the singlebase changes recapitulated the results from studies from the earliest days of molecular biology, but the doublebase changes revealed complex levels of regulation as mutations interacted.

For a more complex test, the authors carried out a similar analysis of three mammalian promoters. Again, the resulting increases or decreases in transcriptional efficiency mostly reproduced those seen in previous publications, but the huge advance is that all of these results came from essentially one experiment. The new technique, dubbed 'synthetic saturation mutagenesis', should prove useful for both gene regulation and synthetic biology studies in the future.

Chris Gunter, HudsonAlpha Institute for Biotechnology

ORIGINAL RESEARCH PAPER Patwardhan, R. P. et al. High-resolution analysis of DNA regulatory elements by synthetic saturation mutagenesis. Nature Biotech. 15 Nov 2009 (doi:10.1038/ nbt.1589)

FURTHER READING Cleary M. A. et al. Production of complex nucleic acid libraries using highly parallel in situ oligonucleotide synthesis. Nature Meth. 1, 241-248 (2004) 